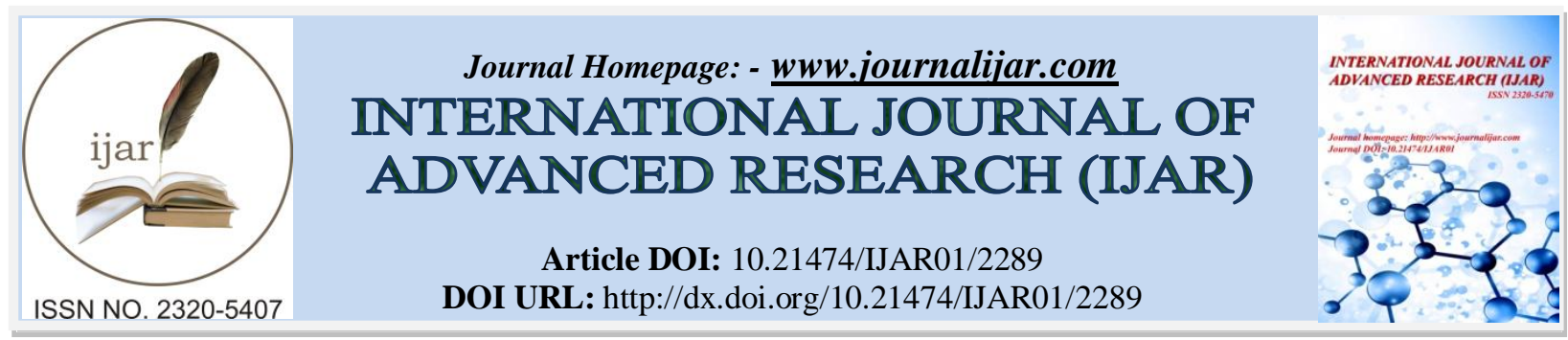

RESEARCH ARTICLE

\title{
SMART HELMET: SMART SOLUTION FOR BIKE RIDERS AND ALCOHOL DETECTION.
}

Snehal Chorge, Hemkedar Kurale, Sonali Deshmukh and Deepa Mane. Department of, Information Technology, APCOER, Pune09.

\section{Manuscript Info}

Manuscript History

Received: 29 September 2016

Final Accepted: 30 October 2016

Published: November 2016

Key words:-

Accident, Drunken Driving, Helmet, Intelligent system.

\begin{abstract}
An accident is an unexpected, unusual, unintended external action which occurs in particular time and place. Carelessness of the driver is the major factor for accident. The government has made rules that rider should compulsory wear the helmet and not consume alcohol and drive. Still the riders do not obey the rules. These accidents are caused due to negligence of the rider. Not wearing the helmet causes the rider with head injuries which may lead to death of the rider. In order to overcome this an intelligent system, smart helmet is proposed, it detects the helmet and also the alcohol present in rider's breath. This system has a pair of transmitter and receiver, the transmitter is placed in the helmet and the receiver is placed at the bike ignition. There are different sensors to ensure the helmet is on the head. These vibration sensors are placed in helmet where the probability of hitting is more. An alcohol sensor is placed near mouth of the rider. The alcohol sensor detects the presence of alcohol in rider's breath. The data of the detection of helmet and alcohol is coded with RF encoder and then transmitted through radio frequency transmitter. The receiver at the bike receives the data and the data is decoded using RF decoder. The result of presence of helmet and the alcohol detection is analyzed on the smart phone. The proposed system will be so designed that if one of the two conditions are violated then also the bike won't start. The bike will start only if the both conditions are followed. This smart helmet will help the rider to compulsory wear helmet and restrict drink and drive condition. MCU controls the function of relay and the ignition, it control the engine through a relay and a relay interfacing circuit.
\end{abstract}

Copy Right, IJAR, 2016,. All rights reserved.

\section{Introduction:-}

An accident is said to be any vehicle accident occurring on a public highway. These accidents therefore include collisions between vehicles and animals, vehicles and pedestrians, or vehicles and fixed obstacles. e to reports the average accidents per day in India are around 1600 and 550 people are dying on each day because of road accidents. The main causes of road accident are drink and drive and not wearing helmet. The usage of helmet by two-wheeler riders is compulsory under Motor Vehicle Act. The section 129 of Motor Vehicle act 1988 makes it must for a rider to wear the helmet. Consumption of alcohol reduces concentration of the rider. It prevents the riders vision due to giddiness. Alcohol obscure fear and actuate the rider to take risks. All above factors causes accidents while driving and many a times it proves dire consequences. The risk of accident doubles for every increase of 0.05 blood alcohol concentration. To make this matter worse Indian traffic officials are not well equipped with the necessary 
equipments required to check. There are laws to check drunken drive and wear helmet but there is no successful implementation of the law. The Motorcycle Act, 1939, has a clause which states that "Motor cycle driven by a drunken rider shall be liable for punishment at first offense for imprisonment for a term of six months or with a fine which may extend two thousand rupees or both for a next offense. The law is very successful if it is made compulsory, but it is usually failed due to the hands of the concerned incharge officer are bribed. The drunken driver is equally to a murderer as he cannot carry out his own tasks without any risk and endanger. These are the two main reasons which motivate us to build the Smart Helmet. The very first step will be detection of the helmet and the alcohol detection. When the both conditions are checked then only the bike ignition will start. IR sensor, PIR sensor and MQ303A alcohol sensors are used for the same. The result obtained from the sensors will be analyzed on the smart phone. This analyzed result will be sent to the concerned authority.

\section{Literature Review:-}

The issue of not wearing the helmet though it is being compulsory and the drink and drive condition to overcome this they proposed a smart helmet. It includes two steps, first is to detect the helmet and the second is to detect the alcohol. When this two conditions are checked then only the bike will start.IR sensor, PIR sensor and MQ-3 alcohol sensor is used for the same. They are using accelerometer to limit the speed of the bike and for fall detection. Fall detection indicates that the accident has occurred. If the fall is detected the message is sent to the bike riders family through GSM. For this accelerometer ADXL335 and GSM module are used.

A simple telemetry system, sensor is activated when pressure that is applied to the helmet's interior when the rider wears it. Once activated the sensor then transmitter sends a control signal to the receiver circuit and activates the relay which is connected to the bikes unit ignition circuit's power supply. The prototype uses a dPDT electromechanical relay for detecting wear the helmet and switching on of the circuit, however on large scale state relays can be out to use which are much faster and have better response.

The smart helmet with radio frequency link, as user wear the helmet a rf signal radiates from transmitter and these rf signals are sensed and synchronized with the help of address matching by the receiver section placed in the ignition switch of the bike and the bike gets started and bike stopped working as the helmet keep out from head. This ensures that the bike works properly till the helmet is on head.

(ITS) Intelligent Traffic System or "Intelligent Vehicle Highway Systems" (IVHS). ITS/IVHS incorporate intelligence in both the roadway infrastructure and in the vehicles with the intention of reducing congestion and environmental impact, and of improving traffic performance, by exploiting the distributed nature of the system and by making use of cooperation and coordination between the various vehicles and the various elements of the roadside infrastructure. IVHS comprise traffic management systems, driver information systems, and vehicle control systems. Automated Highway Systems (AHS) go one step further than IVHS and involve complete automation of the driving task. For better (network-wide) coordination of traffic activities, AHS also distribute the intelligence between the vehicles and the roadside infrastructure.

Identified nine existing safety enhancing ITS systems for motorcycles. In addition, eight emerging technologies currently in prototype form, and several additional 'potential' systems have been described. These have been discussed in terms of the critical motorcycling safety issues, namely loss of control crashes, multiple vehicle crashes, and additional factors such as conspicuity, alcohol and unlicensed riding. While some of these systems serve to address specific safety issues, such as interlocks and alcohol-related crashes, other systems will show comprehensive benefits across a number of crash types. Importantly, this is one area of ITS development that has shown a significant amount of development.

An efficient system of vehicle accident prevention system embedded by alcohol detector. It consist of PIC 16F876A as the main controller, alcohol sensor as the input and three output such as ignition system, LCD display and alarm system. This system capable to alert the driver about the level of drunkenness by indicates the condition on LCD display. It also produce an alarm from buzzer to make the driver aware their own condition and to vigilant other people in surrounding area. The most safety element provided by this system is the driver in high level of drunkenness is not allowed to drive a car as the ignition system will be deactivated. Ultimately, this system help to prevent the driver to drive in risky situation and will avoid accident occur on the road. 
This real time embedded system is based on low cost and easy solution to avoid accidents caused by break of rules and carelessness. The subsystem of the system are ALCOHOL DETECTION-if the rider is found to have drunk alcohol then vehicle does not start, EMERGENCY SYSTEM ACCIDENT- if any movement detected using vibration sensor and using GSM module the area where accident occurs is sent to help center.

A new secret key generation scheme is defined to improve the data security. Secret key generation using received request message (RRM) scheme is used for extraction of secret key. It takes the users request message as input for extraction of secret key. The basic idea is to generate a unique key which can provide data confidentiality and improve the strength of extracted key. This scheme provides high entropy data bit during extraction, thus ensures strength of generated secret key acceptable. Extensive performance evaluation demonstrates that the proposed schemes outperform the existing solutions in terms of highly efficient secret key generation.

Helmet system designed road hazard warning given to rider with wireless bike authentication and traffic adaptive $\mathrm{mp} 3$ playback. The main aim is to provide protection to bike rider and encourage people to wear helmet and to prevent road accidents and follow traffic-rules.

That it checks the wearing of helmet and drunken driving. In this system a safe two wheeler journey is possible which would decrease the head injuries during accidents and also reduce the accident rate due to drunken driving. This system also indicates No parking area which would reduce the crowd of the vehicle in those areas. No entry area is mainly allocated during the construction or repairing of the road, if the rider enters in such area this system would immediately intimate as - No entry area and vehicle will stop automatically. In case of any accident it would send the messages to the friends continuously about the location of the accident happened till the first aid reaches the rider. The system helps to know the location of the vehicle for rescuing in the case of theft incidents.

\begin{tabular}{|l|l|l|}
\hline Sr.No & \multicolumn{1}{|c|}{ Paper Name } & Description \\
\hline 1. & $\begin{array}{l}\text { "Smart Helmet System Using Alcohol } \\
\text { Detection For Vehicle protection" }\end{array}$ & $\begin{array}{l}\text { Detect alcohol consumption, } \\
\text { usage of mobile phones while } \\
\text { driving } \\
\text { SMS or Short Message Service }\end{array}$ \\
\hline 2. & "Intelligent Helmet: Application of RF" & $\begin{array}{l}\text { Radio-frequency identification } \\
\text { (RFID) } \\
\text { UHF radio waves } \\
\text { FID tags }\end{array}$ \\
\hline 3. & $\begin{array}{l}\text { "Brainwave and Alcohol Sensitizing Helmet for } \\
\text { Riders Safety" } \\
\text { Blood Alcohol Content (BAC) } \\
\text { Global Positioning System } \\
\text { (GPS) technology }\end{array}$ \\
\hline 4. & $\begin{array}{l}\text { Safety measures for "Two wheelers by Smart Helmet and Four } \\
\text { wheelers by Vehicular Communication" }\end{array}$ & $\begin{array}{l}\text { GSM and GPS technology. } \\
\text { VANET } \\
\text { RSU }\end{array}$ \\
\hline 5. & $\begin{array}{l}\text { Helmet for Road Hazard Warning with Wireless Bike Authentication } \\
\text { and Traffic Adaptive Mp3 Playback }\end{array}$ & $\begin{array}{l}\text { GPS technology Dashboard } \\
\text { mp3 Player }\end{array}$ \\
\hline 6. & $\begin{array}{l}\text { Intelligent Helmet } \\
\text { Accelerometer }\end{array}$ \\
\hline 7. & $\begin{array}{l}\text { "Smart Helmet Using GSM \&GPS Technology } \\
\text { for Accident Detection and Reporting System" }\end{array}$ & $\begin{array}{l}\text { Alcohol Sensor, } \\
\text { Gsm } \\
\text { Gps } \\
\text { Microcontroller, Pressure Sensor } \\
\text { Vibration Sensor. }\end{array}$ \\
\hline
\end{tabular}

\section{Proposed System:-}

The proposed system mainly focuses on avoidance of drunken driving and restricts to wear the helmet compulsory, which results in Avoidance of accident and save the human life. Now a day's according to government rules this 
smart helmet will be hopefully in use. The proposed system includes Helmet detection, Alcohol detection, and the Android mobile for sending the alert message to person about helmet detection and alcohol detection.

\section{A.1.Helmet Unit:-}

This system will provide helmet sensor switch, infrared sensor switch MCU encoder and Rf transmitter Both the switch \& Alcohol switch is fitted in the helmet unit. The MCU reads data from the sensors \& the sensor gives the result to MCU. If the driver has non-alcoholic breath and also the helmet switch is in a closed position and also it gives the corresponding digital output to RF encoder only. The encoder block will check the conditions are satisfied. it will encode the active inputs to a coded binary output. The RF transmitter will transmit this coded binary output from RF encoder block. The system uses the ASK Modulation technique. In RF transmitter system the digital data is represented in the form of variations in the amplitude of carrier wave by using ASK modulation is known as amplitude shift keying (ASK).

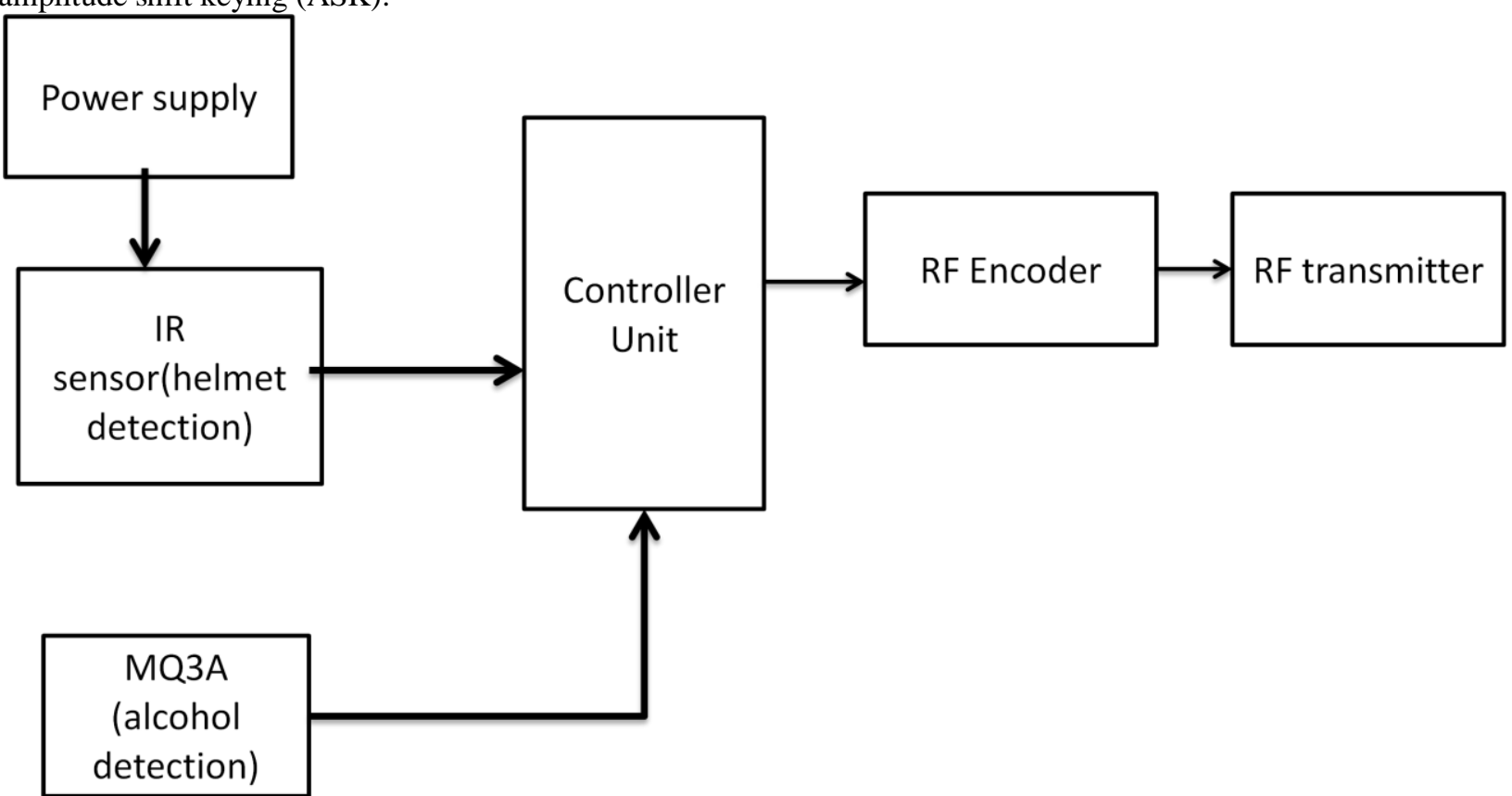

Fig: $\quad$ HELMENT UNIT

\section{A.2.vehicle unit:-}

The vehicle unit includes the RF Receiver, RF Decoder, and MCU. The receiver is the next block of transmitter it receives the coded binary data which is transmitted by the RF transmitter and all data is given to the RF decoder. The RF transmitter gives the input to RF decoder which decodes this input and gives the four bit digital data to the MCU (Micro Controller Unit)only if the address bit of RF encoder \& RF decoder is matches. The MCU block receives the digital data from RF transmitter block. after that it operate the engine of the vehicle, It operates the engine through a relay circuit but it cannot operate the relay directly. So a relay interface is also used here. 


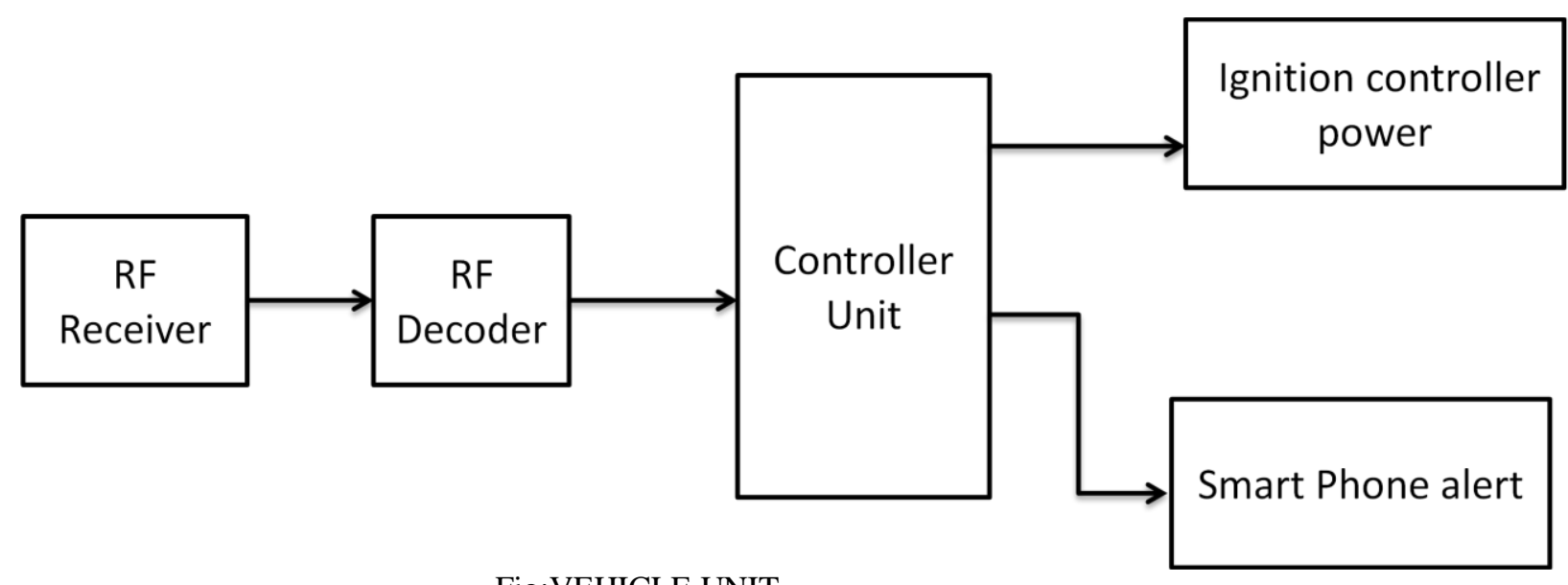

Alcohol detection:-

Fig:VEHICLE UNIT

Now a days we see the people drive the vehicle when they drunk .so this reason the accident is cause so to avoid this accident and save human life the solution is find. This alcohol detection phase the MQ-3 gas detector (Alcohol Sensor)is used for detecting alcohol content from the breath. so the MQ-303A gas detector can be placed below the face defend \& above the additional face detection the surface of sensor is to be sensitive for various alcoholic contractions \& it detects the alcohol from the riders breath. the sensor be sensitive to various alcoholic contraction we programmed threshold limit as $0.04 \mathrm{mg} / \mathrm{L}$ so this system can be integrated with ignition system thus allow to people to handle the vehicle. this sensor is manufactured by Hansel electronic CO-Ltd \& has high sensitivity sensor able to detect BAC with different canentaction

\section{Helmet detection:-}

Most of the people lost their life because not wearing helmet so in proposed system the smart helmet will hopefully used \& can save accident death by $35 \%$ to $45 \%$. the risk of death is 2.5 times more among riders Not wearing a helmet detection of helmet is done using IR \& PIR sensors.

\section{Pir sensor:-}

The PIR Sensor is help to detect if the person wear the helmet or not the PIR(Passive infra-red) sensor it detect the motion by measuring changes in the infrared levels emitted by surrounding objects. this motion of helmet can detected by checking for high signal on a single input output(I/O)Pins At that time bike riders head is detected while he is trying to wear helmet \& check the movement of his head from outside to inside which is give high output used PIR sensor the polo electric devices, such as the PIR sensor which is made of crystalline material that generate an electric charge when exposed to infrared radiation. the changes in the amount of striking and element get change. the voltage generated this change is measure in amplifier board the Fresnel lens which focuses the infrared signals on to the element $\&$ it ambient rapidly in signal changes the on-board amplifier trips output to indicate motion.

\section{Ir sensor:-}

The IR sensor is fitted on the left \& right side of helmet so that human head will be detected. here the IR sensors used the obstacle electrons. the IR LED work as the it transmit the IR signal on to the object \& the signal is to be reflect back from the surface of the helmet this reflected signals are received by an IR receiver \& result is save in the block MCU.

\section{Conclusion:}

The government has taken initiative by making compulsory Helmet and NO Drink and Drive. According to analysis only $10 \%$ bike riders follows these rules. Many a times these rules are violated. The previously developed Helmet only detects the presence of helmet and not the alcohol. The proposed system provides a "Smart Helmet" which detects the alcohol consumed by the rider and whether the rider has worn the helmet or not. This system consists of an android application. The result obtained from the sensors i.e. IR Sensor for Helmet detection and MQ303A for alcohol detection will be analyzed on the smart phone. Hopefully the proposed system will provide the rider's safety and restrict Drink n Drive condition and the traffic rules will also be followed. 


\section{References:-}

1. Sudharsana, Vijayan, Vineed T Govind, Merin Mathews, Simna Surendran, Muhammed Sabah, "Smart Helmet System Using Alcohol Detection For Vehicle protection",International Journal of Emerging Technology in Computer Science \& Electronics (IJETCSE), Volume 8 Issue 1-APRIL 2014.

2. Nitin Agarwal, Anshul Kumar Singh, Pushpendra Pratap Singh, "Intelligent Helmet: Application of RF" International Research Journal of Engineering and Technology (IRJET), Volume: 02 Issue, 02 May-2015

3. Amitava Das, Priti Das, Soumitra Goswami , "Brainwave and Alcohol Sensitising Helmet for Riders Safety" Proceedings of Eleventh IRF International Conference, 17th August 2014.

4. Amitava Das, Priti Das, Soumitra Goswami , "Smart helmet for Indian bike riders "Proceedings of Eleventh IRF International Conference, 17th August 2014.

5. Abhinav Anand, Kumar Harsh, Kushal Kumar, Sourav Gouthi, " Safety measures for "Two wheelers by Smart Helmet and Four wheelers by Vehicular Communication" International Journal of Research in Engineering and Technology, Volume: 04 Issue, 05 May-2015

6. K. Sudarsan, P. Kumaraguru Diderot , “ Intelligent Helmet" international Journal of Science and research(IJOFR) Volume 3 Issue 3, March 2014.

7. www.wikipeia.org/wiki/

8. www.electronicsforu.com

9. www.elecktor.com 